\title{
PERGAMON
}

www.elsevier.com/locate/watres

\section{HYDRODYNAMIC DRAG ON NON-SPHERICAL FLOC AND FREE-SETTLING TEST}

\author{
R. M. WU and D. J. LEE* \\ Department of Chemical Engineering National Taiwan University Taipei, Taiwan,
} 106, R.O.C.

(First received 7 March 2000; accepted in revised form 22 January 2001)

\begin{abstract}
This work numerically investigates the hydrodynamic drag force exerted on a porous spheroid floc moving steadily through a quiescent Newtonian fluid over a wide range of the Reynolds number. The flow patterns for a highly porous spheroid moving at an elevated Reynolds number are basically the same as those at a low Reynolds number, which extends the applicable range of a creeping-flow based correlation to the higher Reynolds number regime. The shape effect becomes more prominent as the spheroid becomes more porous. Using the equivalent diameter, defined as the geometric mean diameter of the principal axes, leads to a universal correlation relating to the drag force, aspect ratio, and interior permeability. In addition, free-settling experiments are performed to estimate how the non-spherical shape affects the three sludge samples. The possible errors in data reduction for the free-settling test are attributed to the $a / b$ ratio and the internal permeability. The errors range from $16-34 \%$ for $a / b=0.6-2.0$. (C) 2001 Elsevier Science Ltd. All rights reserved
\end{abstract}

Key words — drag, spheroid, porous, numerical, free-settling experiment

\section{NOMENCLATURE}

a polar radius, $\mathrm{m}$

$A \quad$ constant in Stokes-law like correlation, dimensionless

$b \quad$ equatorial radius, $\mathrm{m}$

$C_{\mathrm{D}} \quad$ drag coefficient, dimensionless

$d_{\mathrm{f}} \quad$ equivalent diameter of spheroid, $\mathrm{m}$

$F_{\mathrm{D}} \quad$ hydrodynamic resistance of porous sphere, $\mathrm{N}$

$F_{\mathrm{DS}} \quad$ hydrodynamic resistance of porous spheroid, N

$F_{\mathrm{S}} \quad$ hydrodynamic resistance of non-porous sphere, $\mathrm{N}$

$k \quad$ permeability of porous spheroid, $\mathrm{m}^{2}$

$L \quad$ length of circular tube, $\mathrm{m}$

$r \quad$ radial direction, $\mathrm{m}$

$R \quad$ radius of circular tube, $\mathrm{m}$

Re Reynolds number $(2 \rho a V / \mu)$, dimensionless

$V \quad$ velocity of fluid, $\mathrm{m} / \mathrm{s}$

$\mathbf{u}_{\mathrm{f}} \quad$ velocity of the surrounding fluid, $\mathrm{m} / \mathrm{s}$

$\mathbf{u}_{\mathrm{p}} \quad$ fluid velocity within the spheroid, $\mathrm{m} / \mathrm{s}$

$z \quad$ axial direction, $\mathrm{m}$

Greek symbols

$\beta \quad$ dimensionless radius of sphere $(=a / \sqrt{k})$, dimensionless

$\mu \quad$ viscosity of fluid, $\mathrm{Pa} / \mathrm{s}$

$\rho \quad$ density of fluid, $\mathrm{kg} / \mathrm{m}$

$\Omega \quad$ correction factor, dimensionless

*Author to whom all correspondence should be addressed. Tel.: + 886-2-2362-5632; fax: + 886-2-2362-3040; e-mail: djlee@ccms.ntu.edu.tw

\section{INTRODUCTION}

Sufficient information about the hydrodynamic drag force exerted on a highly porous object is necessary when considering its motion, such as sedimentation or centrifugation of sludge flocs, which are highly porous aggregates made of many primary particles (Li and Ganczarczyk, 1989; Jiang and Logan, 1991). Consider a porous sphere moving steadily through a quiescent, infinitely large liquid pool. The drag force exerted on the porous sphere can be stated as follows:

$$
F_{\mathrm{D}}=\left(\pi a^{2}\right)\left(\frac{1}{2} \rho V^{2}\right) C_{\mathrm{D}} \Omega
$$

where $C_{\mathrm{D}}$ is the drag coefficient, $a$ the radius of sphere, $V$ the velocity, $\rho$ the fluid density, and $\Omega$ the ratio of the resistance experienced by the porous sphere to that by an equivalent solid sphere.

Drag coefficient is a function of Reynolds number (Re) and floc sphericity (Clift et al., 1978; Tambo and Watanabe, 1979; Namer and Ganczarczyk, 1993). Under creeping-flow condition $(R e \ll 1), C_{\mathrm{D}}$ for a non-porous sphere is governed by the Stokes law: $C_{\mathrm{D}}=24 / \operatorname{Re}$ and $\Omega=1$. For a highly porous sphere moving steadily through an infinite medium, the corresponding drag force $\left(F_{\mathrm{D}}\right)$ is less than that for a non-porous sphere $\left(F_{\mathrm{S}}\right)$. Restated, $F_{\mathrm{D}}=F_{\mathrm{S}} \Omega$, and $\Omega<1$. Neale et al. (1973) analytically derived the 
correction factor $\Omega$ for a porous sphere under creeping-flow condition. Matsumoto and Suganuma (1977) experimentally verified the theoretical predictions of Neale et al.

Lee et al. (1996) revealed that in many applications involving sludge flocs, the corresponding sphere's $R e$ could reach 40, upon which, the Stokes law would fail. However, previous literature has largely adopted the Stokes law-like correlation (i.e., $C_{\mathrm{D}}=A / R e$, where $A$ is a constant) for satisfactorily interpreting floc settling data beyond the creeping-flow regime (Tambo and Watanabe, 1979; Mitani et al., 1983; Lee and Hsu, 1994; Hung et al., 1996; Chu et al., 1997). This approach, nevertheless, has its limitations. Moreover, the floc interior is highly porous via mass balance calculations with a porosity up to $99 \%$ (Lee, 1994; Wu et al., 1998), which is markedly above the valid limit for the conventionally used Darcy law (Veerapaneni and Wiesner, 1996). Payatakes and Dassios (1987) claimed that the Darcy law combined with Beavers-Joseph-Saffman boundary conditions could approximate flow process over a wide range of porosity values.

At an elevated Reynolds number, the inertial force plays an important role in determining the floc movement, thus yielding nonlinear hydrodynamic governing equations that require numerical solutions. Wu and Lee (1998a) numerically solved the fluid flow field and the associated hydrodynamic drag force exerted on a highly porous sphere moving in an infinite Newtonian fluid at $R e=0.1-40$. Wu and Lee (1998b) considered the case with a porous sphere moving towards an impermeable plate. $\mathrm{Wu}$ and Lee (1999) investigated a porous sphere moving along the centerline of a circular tube. For a highly porous sphere, the above studies confirmed the feasibility of applying the Stokes law-like correlation beyond the creeping-flow regime.

Although previous studies considering the motion of a highly porous object often assumes a perfectly spherical shape, a non-spherical shape characterizes most naturally occurring objects (such as sludge flocs). Other studies (for example, see Le Roux (1997) and the references cited therein) have thoroughly elucidated the characteristics of a non-spherical object by considering the equivalent spherical diameter or sphericity factor and then determining the drag by using an appropriate sphere-based model. Happel and Brenner (1983) derived the analytical solution for the drag force exerted on a non-porous spheroid under creeping-flow condition. Clift et al. (1978) summarized pertinent literature prior to 1978. Later, Chhabra (1995) provided experimental data for non-porous, non-spherical particles moving beyond a creeping-flow regime. Brenner (1996) reviewed the approaches applied to the Stokes hydrodynamic resistance of non-spherical particles. Zlatanovski (1999) investigated the creeping flow past a porous prolate spheroidal particle using the Brinkman model.
The non-spherical shape of sludge flocs has implications for the free-settling test. Nevertheless, the hydrodynamic drag force of a highly porous spheroid moving at an elevated Reynolds number $(>1)$ has seldom been investigated. This work numerically investigates the fluid flow field and the hydrodynamic drag force exerted on a moving porous spheroid at a Reynolds number ranging from 0.1 to 40 . The extent to which shape affects the drag force is also discussed. Moreover, free-settling experiments on three sludge samples, i.e., a waste activated sludge and two cupric hydroxide sludges, are performed to investigate the implications of shape effects to data reduction and interpretation.

\section{GOVERNING EQUATIONS AND SOLUTION}

Consider a porous spheroid with principal-axis radii of $a$ and $b$, whose internal permeability is $k$, moving along its axis of revolution at a steady speed of $V$ through an infinite, quiescent Newtonian fluid of viscosity $\mu$ and density $\rho$. The combined DarcyBrinkman model describes the flow field $\mathbf{u}_{\mathrm{p}}$ within the porous spheroid; meanwhile, the surrounding Newtonian fluid field, $\mathbf{u}_{\mathrm{f}}$, is governed by the steadystate Navier-Stokes equations. To easily define the boundary conditions in the computational domain, an equivalent problem is considered: the spheroid is fixed at the centerline while the surrounding fluid is flowing at a uniform speed of $V$ from infinity towards the fixed spheroid. Wu and Lee (1998a) provides the related boundary conditions. Figure 1 illustrates the process along with the governing equations.

The governing equations are solved together with the boundary conditions using the general-purpose computational fluid dynamics program FIDAP 7.5 (FDI Inc., USA). The number of elements in the fluid side and within the porous spheroid is about 18,000 and 30, respectively. Notably, the fluid field within and around the porous spheroid is of major concern, accounting for why more elements are allocated near

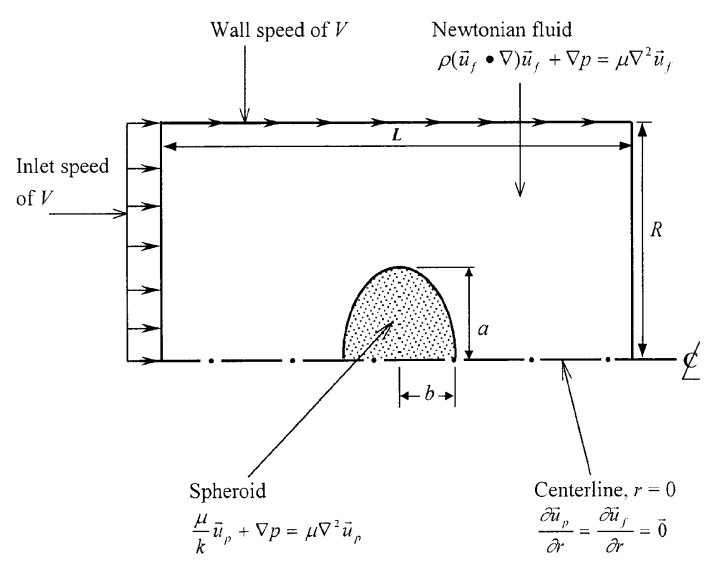

Fig. 1. Computation domain under investigation. 
the spheroid to increase accuracy. The maximum relative error of $10^{-3}$ can be achieved by three to five iterations. FIDAP evaluates the hydrodynamic drag force on the porous spheroid $\left(F_{\mathrm{DS}}\right)$ with its postprocessing program FIPOST (FIDAP, 1993).

\section{NUMERICAL CALCULATIONS}

\section{Fluid flow fields}

Figures 2 and 3 plot the streamline and velocity field for $a / b=1.43$ and $0.7, \beta=0.5$ (highly porous spheroid) or infinity (nonporous spheroid) at $R e$ of 0.1 or 40 , respectively. Where parameter $\beta$ is the dimensionless polar radius, defined as $a / k^{0.5}$. Comparing these figures reveals the effects of aspect ratio $(a / b)$, permeability, and Reynolds number on the flow field. As expected, all fluid flow characteristics for the porous sphere demonstrated in $\mathrm{Wu}$ and Lee (1998a, b) are also noted in cases for porous spheroid, which are briefly discussed as follows.

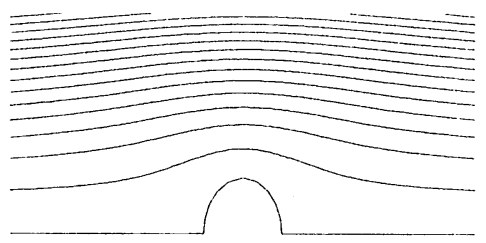

(a) $a / b=1.43, \beta \rightarrow \infty, \operatorname{Re}=0.1$

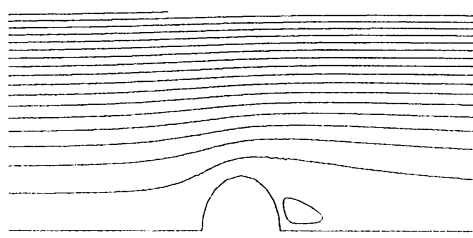

(c) $a / b=1.43, \beta \rightarrow \infty, R e=40$

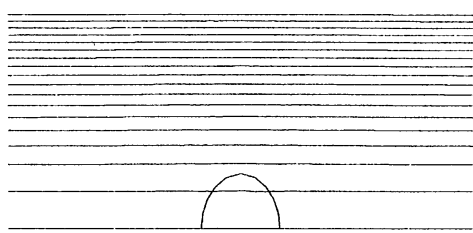

(e) $a / b=1.43, \beta=0.5, R e=0.1$

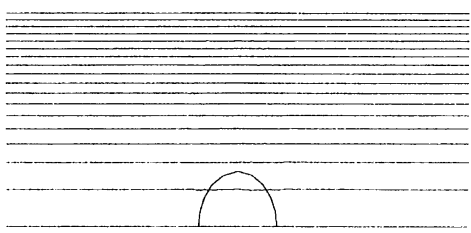

(g) $a / b=1.43, \beta=0.5, R e=40$
For non-porous spheroids, the originally flatshaped flow field is markedly distorted when approaching the spheroid, as attributed to the noslip boundary condition that the fluid velocity drops to zero on the solid surface. At $R e=0.1$, the streamlines move mainly along (parallel to) the spheroid surface, and are nearly symmetrical in the front and the rear sides of the spheroid (Figs 2(a) and 2(b)). The velocity field reveals a similar feature (Figs 3(a) and 3(b)). The process is termed hereinafter as "viscous-controlled".

At elevated $R e$, the boundary layer separation, accompanied with asymmetric fluid flow patterns and a wake region, appears behind the spheroid. Notably, the actual streamlines for a floc moving steadily in a pool could be obtained simply by adding a fluid velocity $-V$ onto the ones illustrated in Fig. 2. Comparing Figs 2(b) and 2(d) reveals an essential flow pattern difference. Owing to that the fluid does not flow parallel to the solid surface, the inertia force has come into play and, together with the viscous force, dominates the flow process.

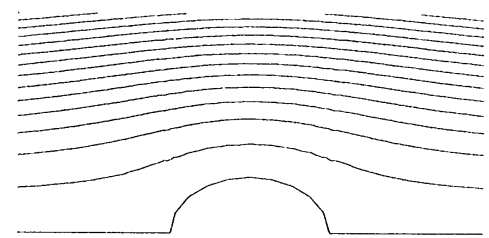

(b) $a / b=0.7, \beta \rightarrow \infty, R e=0.1$

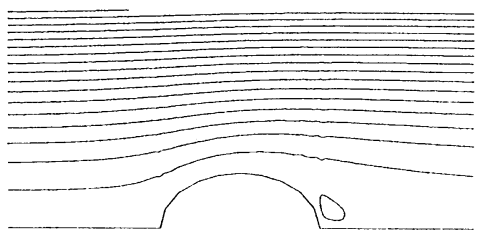

(d) $a / b=0.7, \beta \rightarrow \infty, R e=40$

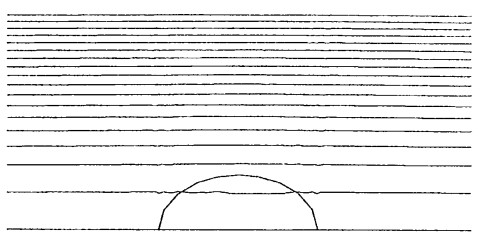

(f) $a / b=0.7, \beta=0.5, R e=0.1$

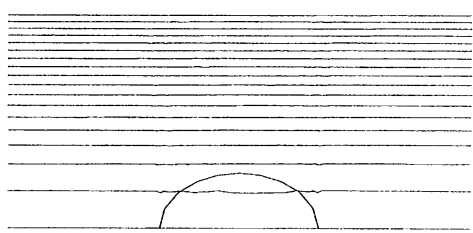

(h) $a / b=0.7, \beta=0.5, R e=40$

Fig. 2. Calculated streamline plots. 


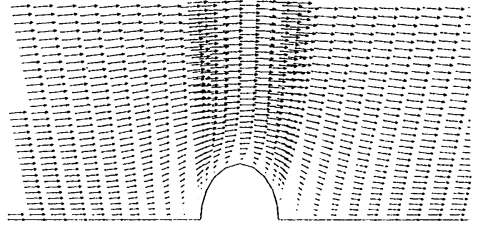

(a) $a / b=1.43, \beta \rightarrow \infty, R e=0.1$

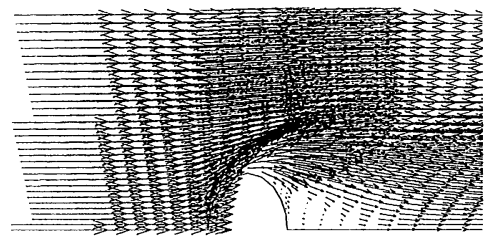

(c) $a / b=1.43, \beta \rightarrow \infty, R e=40$

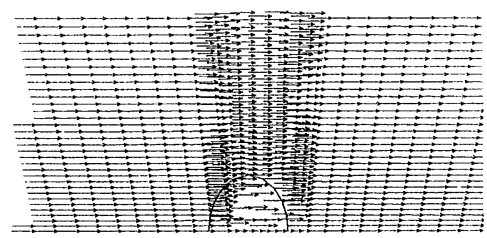

(e) $a / b=1.43, \beta=0.5, R e=0.1$

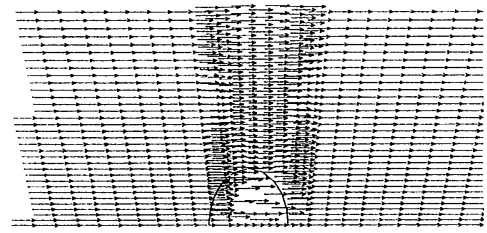

(g) $a / b=1.43, \beta=0.5, R e=40$

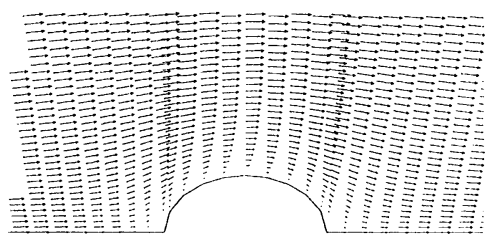

(b) $a / b=0.7, \beta \rightarrow \infty, R e=0.1$

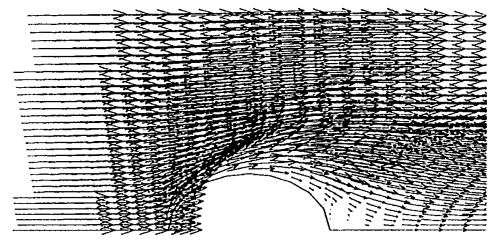

(d) $\alpha / b=0.7, \beta \rightarrow \infty, R e=40$

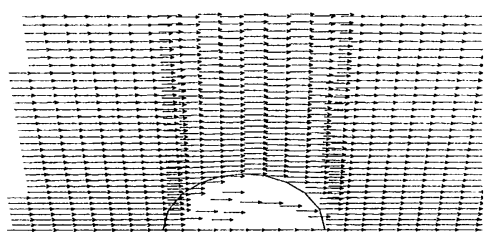

(f) $a / b=0.7, \beta=0.5, R e=0.1$

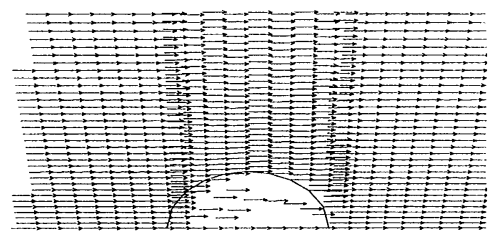

(h) $a / b=0.7, \beta=0.5, R e=40$

Fig. 3. Calculated fluid velocity plots.

Nevertheless, for a highly porous spheroid, the fluid can not only flow around, but also flow through the porous spheroid. At $R e=0.1$, the streamlines remain nearly flat-shaped when passing through the porous spheroid (Figs 2(e) and 2(f)). Except along the central line of the spheroid interior, the fluid velocity decreases (which is not clearly ascertainable in Figs 2(e) and 2(f)), the fluid velocity remains almost uninfluenced by the porous spheroid. A similar pattern is noted at $R e=40$ (Figs 2(g)-(h) and $3(\mathrm{~g})-(\mathrm{h}))$, except that the corresponding velocity has become higher than that at $R e=0.1$. (Note: These two figures resemble each other while become hard to differentiate with. Nevertheless, the velocity vectors are in different scale, with those at $R e=40$ much greater than those at $R e=0.1$.) This observation closely corresponds to that of Wu and Lee (1998a, b) for a porous sphere that, owing to the advection flow through the spheroid interior, the boundary layer separation and the after-spheroid wake do not occur regardless of the Reynolds number. Li and Ganc- zarczyk $(1988,1992)$ also found the advection flow in a moving floc.

\section{Drag force}

The drag force exerted on the sphere can be numerically evaluated, subsequently leading to the correction factor $\Omega\left(F_{\mathrm{DS}} / F_{\mathrm{S}}\right)$. Where $F_{\mathrm{S}}$ is the drag force exerted on the sphere of radius $a$. Table 1 lists the calculation results. Some efforts had been devoted to confirm the accuracy of computational results. A comparison is made with literature with respect to the non-porous cases $(\beta=\infty)$ over a wide range of Re (Happel and Brenner, 1983). Generally, the calculation results correlate well with literature with a relative error less than $3 \%$.

Figure 4 presents the correction factor $\Omega$ for $a / b=$ 1.43 (oblate spheroid) as an illustrative example. The $\Omega-\beta$ relationships at different $a / b$ closely resemble each other and are neglected for simplicity. The $\Omega-\beta$ curves exhibit an S-shape characteristic; meanwhile, 
Table $1 . \Omega$ value as a function of $R e$ and $\beta$.

\begin{tabular}{|c|c|c|c|c|c|c|c|}
\hline$a / b=2.5$ & $R e=0.1$ & 1 & 5 & 10 & 20 & 30 & 40 \\
\hline$\beta=5$ & 0.494 & 0.483 & 0.453 & 0.435 & 0.391 & 0.369 & 0.350 \\
\hline 2.24 & 0.228 & 0.218 & 0.184 & 0.160 & 0.127 & 0.110 & 0.099 \\
\hline 1.58 & 0.138 & 0.130 & 0.105 & 0.089 & 0.068 & 0.059 & 0.052 \\
\hline 0.5 & 0.017 & 0.016 & 0.012 & 0.010 & 0.007 & 0.006 & 0.005 \\
\hline \multicolumn{8}{|l|}{$a / b=1.67$} \\
\hline$\beta=5$ & 0.618 & 0.607 & 0.579 & 0.566 & 0.524 & 0.506 & 0.490 \\
\hline 2.24 & 0.325 & 0.312 & 0.271 & 0.242 & 0.198 & 0.175 & 0.160 \\
\hline 1.58 & 0.207 & 0.197 & 0.163 & 0.141 & 0.111 & 0.096 & 0.086 \\
\hline 0.5 & 0.028 & 0.026 & 0.020 & 0.016 & 0.012 & 0.011 & 0.009 \\
\hline \multicolumn{8}{|l|}{$a / b=1.43$} \\
\hline$\beta=5$ & 0.648 & 0.637 & 0.609 & 0.595 & 0.552 & 0.534 & 0.520 \\
\hline 2.24 & 0.357 & 0.344 & 0.300 & 0.269 & 0.221 & 0.197 & 0.180 \\
\hline 1.58 & 0.232 & 0.221 & 0.184 & 0.160 & 0.126 & 0.109 & 0.098 \\
\hline 0.5 & 0.032 & 0.030 & 0.023 & 0.019 & 0.014 & 0.012 & 0.011 \\
\hline \multicolumn{8}{|l|}{$a / b=1.25$} \\
\hline$\beta=5$ & 0.688 & 0.677 & 0.652 & 0.640 & 0.598 & 0.582 & 0.569 \\
\hline 2.24 & 0.394 & 0.380 & 0.335 & 0.303 & 0.252 & 0.226 & 0.207 \\
\hline 1.58 & 0.261 & 0.249 & 0.210 & 0.183 & 0.146 & 0.127 & 0.115 \\
\hline 0.5 & 0.037 & 0.035 & 0.027 & 0.022 & 0.017 & 0.014 & 0.013 \\
\hline \multicolumn{8}{|l|}{$a / b=1$} \\
\hline$\beta=5$ & 0.691 & 0.683 & 0.657 & 0.639 & 0.630 & 0.633 & 0.620 \\
\hline 2.24 & 0.449 & 0.435 & 0.388 & 0.353 & 0.302 & 0.265 & 0.244 \\
\hline 1.58 & 0.278 & 0.265 & 0.221 & 0.190 & 0.163 & 0.153 & 0.139 \\
\hline 0.5 & 0.046 & 0.043 & 0.034 & 0.028 & 0.022 & 0.018 & 0.016 \\
\hline \multicolumn{8}{|l|}{$A / b=0.8$} \\
\hline$\beta=5$ & 0.809 & 0.802 & 0.780 & 0.767 & 0.719 & 0.703 & 0.690 \\
\hline 2.24 & 0.516 & 0.502 & 0.453 & 0.416 & 0.352 & 0.320 & 0.296 \\
\hline 1.58 & 0.363 & 0.349 & 0.300 & 0.266 & 0.215 & 0.190 & 0.172 \\
\hline 0.5 & 0.058 & 0.054 & 0.042 & 0.035 & 0.027 & 0.023 & 0.020 \\
\hline \multicolumn{8}{|l|}{$A / b=0.7$} \\
\hline$\beta=5$ & 0.851 & 0.843 & 0.821 & 0.806 & 0.752 & 0.734 & 0.719 \\
\hline 2.24 & 0.557 & 0.543 & 0.493 & 0.454 & 0.386 & 0.350 & 0.325 \\
\hline 1.58 & 0.398 & 0.384 & 0.332 & 0.295 & 0.240 & 0.212 & 0.192 \\
\hline 0.5 & 0.066 & 0.062 & 0.048 & 0.040 & 0.031 & 0.026 & 0.023 \\
\hline \multicolumn{8}{|l|}{$a / b=0.6$} \\
\hline$\beta=5$ & 0.903 & 0.897 & 0.874 & 0.857 & 0.797 & 0.776 & 0.759 \\
\hline 2.24 & 0.609 & 0.595 & 0.544 & 0.503 & 0.429 & 0.391 & 0.363 \\
\hline 1.58 & 0.443 & 0.428 & 0.374 & 0.333 & 0.272 & 0.241 & 0.219 \\
\hline 0.5 & 0.076 & 0.072 & 0.056 & 0.047 & 0.036 & 0.030 & 0.027 \\
\hline \multicolumn{8}{|l|}{$a / b=0.5$} \\
\hline$\beta=5$ & 0.977 & 0.972 & 0.950 & 0.930 & 0.862 & 0.836 & 0.816 \\
\hline 2.24 & 0.679 & 0.665 & 0.612 & 0.568 & 0.487 & 0.445 & 0.413 \\
\hline 1.58 & 0.504 & 0.488 & 0.430 & 0.385 & 0.316 & 0.280 & 0.255 \\
\hline 0.5 & 0.091 & 0.085 & 0.067 & 0.056 & 0.043 & 0.037 & 0.032 \\
\hline
\end{tabular}

$\Omega$ approaches zero at $\beta<0.5$ (lower plateau; $=0$ ) or at $\beta>40$ (upper plateau; $=0.92$ ). The upper plateau value at $R e=0.1$ correlates with Happel and Brenner (1983) (0.941), which partially verifies the validity of present numerical results. Consequently, the porous spheroid at $\beta>40$ could be taken as a non-porous object. Both increasing $R e$ and/or decreasing $\beta$ leads to a lower $\Omega$. The $\beta$ range within which $R e$ is mostly affecting $\Omega$ is between 0.5 and 10 . For example, at $\beta=5$, the $\Omega$ value is 0.648 or 0.520 at $R e=0.1$ or 40 , respectively. At $\beta=0.5$, the corresponding $\Omega$ values are 0.0321 and 0.0108 , respectively. Wu et al. (1998a, b) estimated the $\beta$ values for activated sludge floc, a naturally occurring porous spheroid, as ranging from 1.4 to 6.8 . Consequently, it is a difficult task to use the $\Omega$ value obtained for creeping-flow condition in interpreting the free-settling data of sludge flocs over a wide range of $R e$, as usually adopted in literature.
At $R e=0.1$, the corresponding upper plateau $\Omega$ value at $a / b=0.7$ is 1.084 , which is greater than that for $a / b=1.43$. The reasons corresponding to the larger plateau value for a prolate spheroid than those for an oblate spheroid are self-evident. Again, this value correlates well with that listed in Happel and Brenner (1983) (1.087). Moreover, the relative error is less than $0.28 \%$.

\section{Effects of nonspherical shape}

According to equation (1) the product of $C_{\mathrm{D}} \Omega$ controls the drag force. Figure 5 plots the numerical values of the product of $C_{\mathrm{D}} \Omega\left(F_{\mathrm{DS}} /\left(\pi a^{2} \rho V^{2} / 2\right)\right)$ against the spheroid's $\operatorname{Re}(=2 \rho a \mathrm{~V} / \mu)$ at $\beta=1.58$ and 0.5 , respectively, with $a / b$ as a parameter. All curves are a straight line of slope -1 on a $\log -\log$ scale. This finding suggests that the Stokes-law like correlation can be extended from creeping-flow 


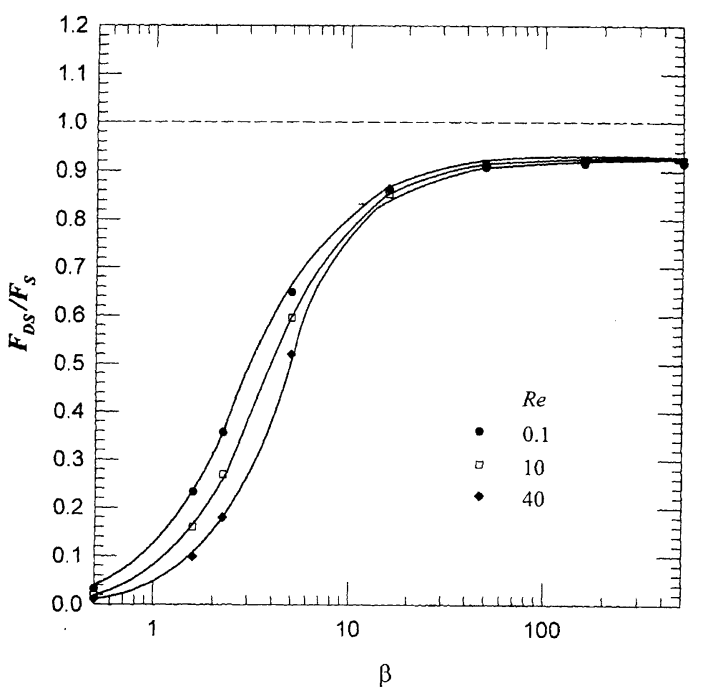

Fig. 4. $F_{\mathrm{DS}} / F_{\mathrm{S}}$ vs. $\beta$ plot. $a / b=1.43$.

regime to that at a high $R e$. Furthermore, at a prescribed $R e$, the $C_{\mathrm{D}} \Omega$ decreases with an increasing $a / b$. This corresponds to the general conclusions that the drag on a prolate spheroid is greater than that for an oblate spheroid.

Shape effect becomes more prominent at a reduced $\beta$ and higher Re. Consider two porous spheroids whose $\beta=1.58$ to be at the same $a$ but at different values of $a / b$ of 0.6 and $1 / 0.6$. According to Table 1 , the relative error in estimating their drag forces at $R e=0.1$ and $\beta=1.58$, based on the sphere of radius $a$ while neglecting the shape effect, are approximately of 60 and $25 \%$, respectively. For example, $\Omega=0.443$ and 0.278 at $a / b=0.6$ and 1 , respectively. Hence the relative error for drag force estimation is $(0.278$ $0.443) / 0.278=-60 \%$. At $R e=10$, the corresponding relative errors become 76 and $26 \%$, respectively. Next, consider two porous spheroids whose $\beta=0.5$. The relative errors at $R e=0.1$ are 65 and $40 \%$; while at $R e=10$, are 68 and $43 \%$, respectively.

The ratio of $C_{\mathrm{D}} \Omega$ in Fig. 5 is evaluated based on polar radius $a$. The equivalent diameter, defined as $d_{\mathrm{f}}=2 \sqrt{a b}$, the geometric mean of the two radii, was frequently employed instead of $a$ (Hung et al., 1996; Chu et al., 1997). Figure 6 depicts the $C_{\mathrm{D}} \Omega$ data evaluated based on $d_{\mathrm{f}} / 2$ rather than on $a$. (That is, $C_{\mathrm{D}} \Omega=F_{\mathrm{DS}} /\left(\pi d_{\mathrm{f}}^{2} \rho V^{2} / 8\right)$. However, $R e$ is still based on polar radius $a$. When $\beta$ becomes small, all data fall onto a single curve that depends only on $\beta$. Restated, the following expression is approximately valid for a highly porous spheroid:

$$
C_{\mathrm{D}} \Omega\left(\text { based on } d_{\mathrm{f}}\right)=A(\beta) / \operatorname{Re} \quad(\text { based on } a)
$$

Equivalently, equation (1) could be stated as follows:

$$
F_{\mathrm{DS}}=\frac{A(\beta)}{4} \pi \mu V b
$$

Equation (3) is valid for a highly porous spheroid moving at a Reynolds number from less than 0.1 to

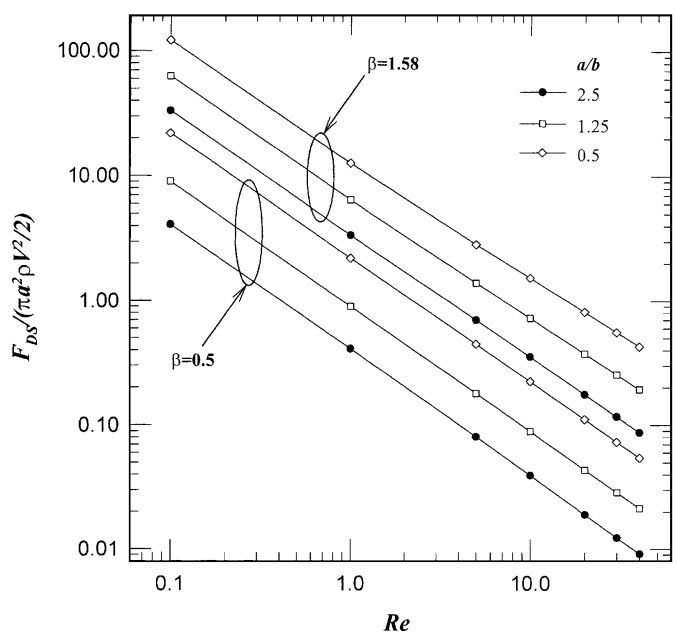

Fig. 5. $F_{\mathrm{DS}} /\left(\pi a^{2} \rho V^{2} / 2\right)$ against $R e$ plot for highly porous spheroids.

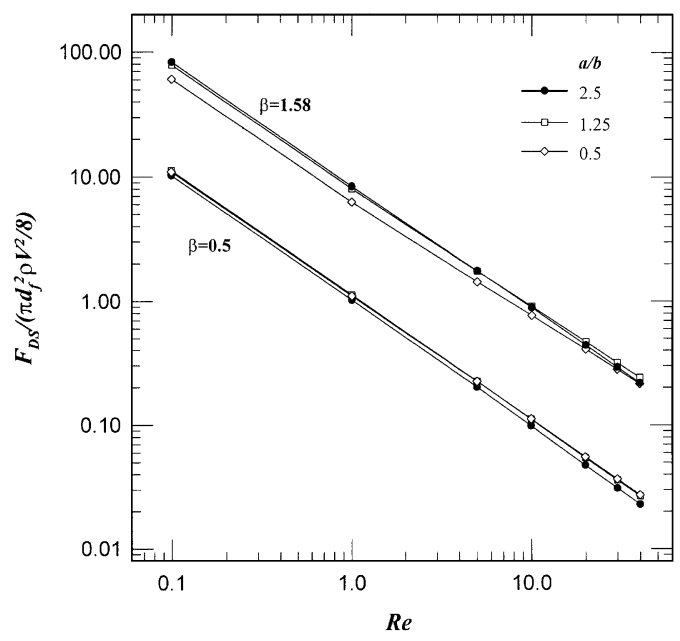

Fig. 6. $F_{\mathrm{DS}} /\left(\pi d_{\mathrm{f}}^{2} \rho V^{2} / 8\right)$ against $R e$ plot for highly porous spheroids. $R e$ is based on the polar diameter $2 a$.

40. The corresponding $A$ values at various $R e$ can be found by allowing $R e=1$ in Fig. 6. For example, at $\beta=0.5$ and 1.58 , the corresponding $A$ values are 1 and 10 , respectively. If at $a \rightarrow b$ and $\beta \rightarrow \infty, A \rightarrow 24$, then equation (3) reduces to the conventional Stokes law.

Over the $\beta$ range in which equation (3) becomes valid, Fig. 7 illustrates the linear correlation between $A(\beta)$ and $\beta$ value. A simple equation $A(\beta)=6 \beta-2$ describes the dependence for $0.5<\beta<1.58$.

\section{EXPERIMENTS}

Samples and tests

We herein examined three sludge samples. A waste activated sludge sample was taken from the wastewater treatment plant in Neili Bread Plant, Presidental Enterprise Co., Taoyuan, Taiwan, and was tested within $2 \mathrm{~h}$ after 


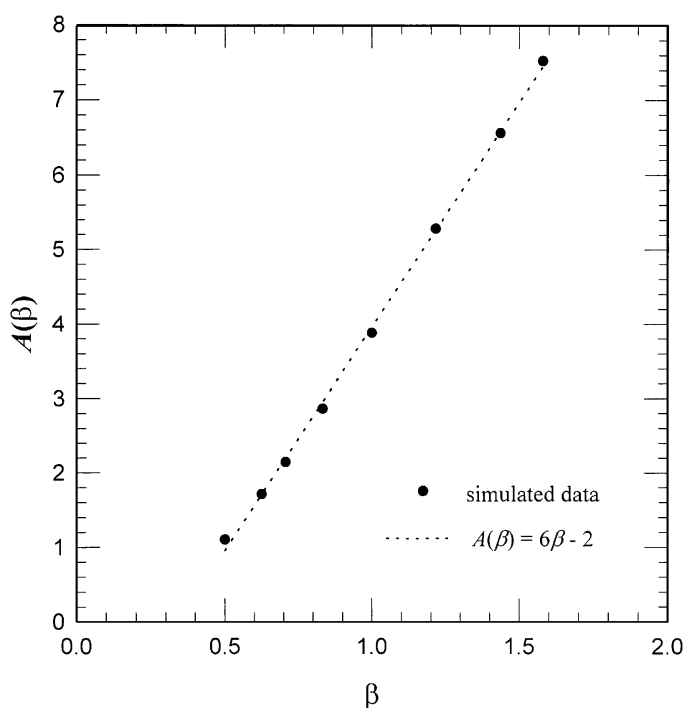

Fig. 7. Correlation between $A(\beta)$ and $\beta$ values for highly porous spheroids.

sampling. The chemical oxygen demand (COD), suspended solids (SS) and turbidity data were for the supernatant drawn from the sludge, determined using EPA Taiwan standard methods. The results read: $5.6 \mathrm{mg} / \mathrm{L}$ (COD), $7.1 \mathrm{mg} / \mathrm{L}$ (SS) and $1.39 \mathrm{NTU}$ (turbidity). The weight percentage of the sludge sample was $0.7 \%(\mathrm{w} / \mathrm{w})$.

Cupric hydroxide sludge sample is prepared by mixing $50 \mathrm{~g} \mathrm{CuSO}_{4} \cdot 5 \mathrm{H}_{2} \mathrm{O}$ and $18 \mathrm{~g} \mathrm{NaOH}$ with $950 \mathrm{~g}$ distilled water. All chemicals are of GR grade from Merck Co. and were used as supplied. To illustrate the fluid shear effect, a Couette-flow reactor, which consists of two coaxial cylinders having a length of $30 \mathrm{~cm}$, an inner diameter of $8 \mathrm{~cm}$ and an outer diameter of $9 \mathrm{~cm}$, was employed for shear experiments. The original cupric hydroxide sludge was poured into the reactor whose inner cylinder rotated at $1000 \mathrm{rpm}$ for $20 \mathrm{~min}$. After shearing samples were drawn carefully from the reactor to test.

A glass cylinder $(6 \mathrm{~cm}$ in diameter and $50 \mathrm{~cm}$ in height $)$, sectioned on a side with an attaching plane view glass, was used for floc-settling test. A JAI 950 camera equipped with a close-up lens was used to record the floc motion. The two principal-axis radii of the floc were measured with the assistance of software Inspector (Matrox), denoted as radius $a$ and $b$. By defining the mass center, the location versus time data of the floc could be traced and recorded. The floc moving speed could be found by numerical differentiation and data smoothing. The ultimate error for length measurement was determined by the optical resolution of the camera system, estimated within $1 \%$. From the distance versus time data the floc moving speed can be found by numerical differentiation and data smoothing. The maximum error in velocity estimation is within $2 \%$.

\section{RESULTS}

Figure 8 depicts some terminal velocity versus floc diameter $\left(d_{\mathrm{f}}\right)$ data, indicated with their $a / b$ ratios. The waste activated sludge flocs exhibits a more irregular shape compared with the cupric hydroxide slurries. As Fig. 8 depicts, the terminal velocity of flocs increases with $d_{\mathrm{f}}$, a self-evident result. The experimental data clearly fall into three groups for

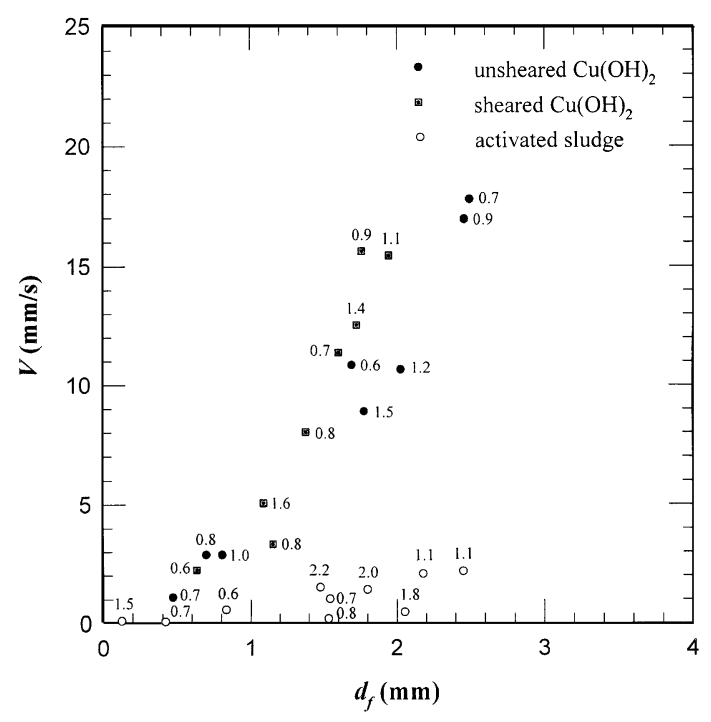

Fig. 8. Typical experimental terminal velocity vs. floc diameter data. Numerical values are for $a / b$ ratios.

the three sludges used. However, large data scattering reveals the possible role of the non-spherical shape. Based on the same floc diameter, the velocity data follow the sequence: sheared cupric hydroxide $>$ unsheared cupric hydroxide $>$ activated sludge. The investigated Reynolds number ranges for the former two sludges are hence much greater than that for the latter. The experimental range for activated sludge includes: $0.005<R e<2.9$, and $0.6<a / b<2.2$. The corresponding ranges for sheared and unsheared cupric hydroxide sludges are $0.5<R e<29$, and $0.5<a / b<1.6$, and $0.2<\operatorname{Re}<20$, and $0.7<a / b<2.2$, respectively.

\section{Drag force estimation}

In literature works the floc in the free-settling tests is commonly assumed spherical. Based on the force balance for floc motion the density of floc (and the porosity as well) is subsequently estimated (Lee et al., 1996). Numerical simulations were carried out floc by floc based on the corresponding measured $a, b$, and $V$. These experimental data were used in numerical simulations. Wu et al. (1999) estimated the $\beta$ values for the activated sludge and cupric hydroxide sludge (sheared and unsheared) are approximately 2.5 and 10 , respectively, which were adopted in the present simulations. (Note: The permeability of flocs should be a function of floc size. However, over a rather wide range of floc size, the floc permeability was noted to increase proportionally with the square of floc size, hence yielding a nearly constant $\beta$ value for a specific sludge floc (Gmachowski, 1999)).

Two sets of simulations were conducted for each floc settling test: the first for a porous spheroid of measured principal-axis radii of $a$ and $b$, and the second for a porous sphere of $d_{\mathrm{f}}(2 \sqrt{a b})$. The 


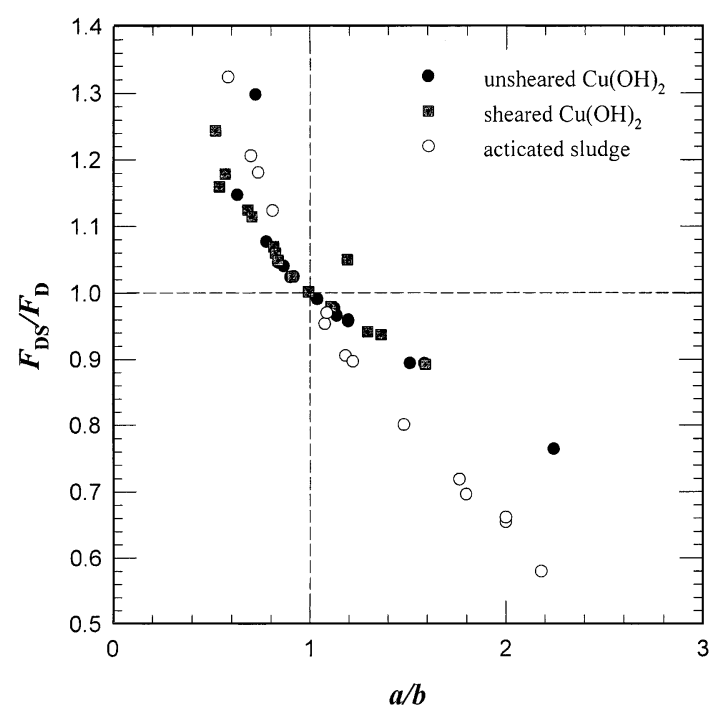

Fig. 9. $F_{\mathrm{DS}} / F_{\mathrm{D}}$ ratio vs. $a / b$ plot.

corresponding calculated drag forces were denoted as $F_{\mathrm{DS}}$ and $F_{\mathrm{D}}$, respectively. To interpret the freesettling data requires the drag forces to floc motion. The ratio $F_{\mathrm{DS}} / F_{\mathrm{D}}$ thereby indicates the error range to assume the floc as a sphere in free-settling test. A ratio deviating more readily from unity indicates a greater error might be introduced in interpreting freesettling data.

Figure 9 illustrates the $F_{\mathrm{DS}} / F_{\mathrm{D}}$ versus $a / b$ relationship for the three sludge samples. Regardless of the sludge species, the three sets of results closely correspond with each other. Apparently, $F_{\mathrm{DS}} / F_{\mathrm{D}}=1$ when $a=b$. When $a / b$ deviates from unity, the drag force exerted on a spheroid would differ from that of an equivalent sphere. The possible errors might be introduced in data reduction for free-settling test depending upon the $a / b$ ratio and the internal permeability. For example, for $a / b=0.6$ (prolate spheroid), $F_{\mathrm{DS}} / F_{\mathrm{D}}=1.33$ for activated sludge $(\beta=$ $2.5)$ and $=1.20$ for cupric hydroxide $(\beta=10)$. Restated, the drag force exerted on an "elongated" floc with $a=0.6 b$ would be underestimated by 16-33\%. On the other hand, for a "flattened" floc with $a=2 b$ (oblate spheroid), the exerted drag force would be overestimated by $20-34 \%$ to assume a spherical shape.

\section{CONCLUSIONS}

This work numerically investigates the hydrodynamic drag force exerted on an individual spheroid moving steadily through a quiescent Newtonian fluid over a Reynolds number ranging from 0.1 to 40 . The spheroid, with $a$ and $b$ as the two principal-axis radii, is assumed to be highly porous and is moving along the axis of revolution. At the ratio, $a / b$, ranging from
0.5 to 2.5 , the computational fluid dynamics software, FIDAP 7.5, numerically solves the fluid fields within and around the moving spheroid, from which the corresponding hydrodynamic drag force is subsequently evaluated. The flow patterns for a highly porous spheroid moving at low or elevated Reynolds number are basically the same. Both the increase in Reynolds number, decrease in $\beta$ and increase in the ratio of principal-axis radii $(a / b)$ lead to a reduction in correction factor $\Omega$. Moreover, shape effects of highly porous spheroid become more significant when $\beta$ decreases. Employment of the equivalent diameter, defined as the geometric mean diameter of the principal-axis radii, leads to a universal correlation.

Free-settling experiments are also conducted for three sludge samples: a waste activated sludge and two cupric hydroxide sludges. The possible errors might be introduced in data reduction for freesettling test, depending on the $a / b$ ratio and the internal permeability. The drag force exerted on an "elongated" floc with $a=0.6 b$ is underestimated by $16-33 \%$. On the other hand, for a "flattened" floc with $a=2 b$, the exerted drag force is overestimated by $20-34 \%$ to assume a spherical shape.

Acknowledgements - This work is supported by National Science Council, ROC.

\section{REFERENCES}

Brenner H. (1996) The Stokes hydrodynamic resistance of nonspherical particles. Chem. Engng. Commun. 148, 487-562.

Chhabra R. P. (1995) Wall effects on free-settling velocity of non-spherical particles in viscous media in cylindrical tubes. Powder Technol. 85, 83-90.

Chu C. P., Feng W. H., Tsai I. H. and Lee D. J. (1997) Unidirectional freezing of waste activated sludge: The presence of sodium chloride. Environ. Sci. Technol. 31, $1512-1517$.

Clift R., Grace J. R. and Weber M. E. (1978) Bubbles, Drops and Particles. Academic Press, NY.

FIDAP (1993) FIPOST Users Manual, Fluid Dynamics Int., Inc. IL, USA, 56-1.

Gmachowski, L. (1999) Comments to hydrodynamic drag force exerted on a moving floc and its implications to free settling test. Water Res. 32, 860-868; Water Res. 33, 1114-1115.

Happel J. and Brenner H. (1983) Low Reynolds Number Hydrodynamics. Table 4-26.1, p. 149, Academic Press, NY.

Hung W. T., Chang I. L., Chen G. W. and Lee D. J. (1996) Uni-directional freezing of waste activated sludge: The effects of freezing speed. Environ. Sci. Technol. 30, 2391-2396.

Jiang Q. and Logan B. E. (1991) Fractal dimensions of aggregates determined from steady-state size distribution. Environ. Sci. Technol. 25, 2031-2038.

Le Roux J. P. (1997) Comparison of sphericity indices as related to the hydraulic equivalence of settling grains. J. Sed. Res. 67, 527-530.

Lee D. J. (1994) Floc structure and bound water content in excess activated sludges. J. Chem. Ind. Chem. Engng. 25, 201-207. 
Lee D. J., Chen G. W., Liao Y. C. and Hsieh C. C. (1996) Use of free-settling test to estimate activated sludge flocs density. Water Res. 30, 541-550.

Lee D. J. and Hsu Y. H. (1994) Fast freeze/thaw treatment on activated sludge: Floc structure and sludge dewaterability. Environ. Sci. Technol. 28, 1444-1449.

Li D. H. and Ganczarczyk J. J. (1988) Flow through activated sludge flocs. Water Res. 22, 789-792.

Li D. H. and Ganczarczyk J. J. (1989) Fractal geometry of particle aggregates generated in water and wastewater treatment process. Environ. Sci. Technol. 23, $1385-1389$

Li D. H. and Ganczarczyk J. J. (1992) Advective transport in activated sludge flocs. Water Environ. Res. 64, 236-240.

Matsumoto K. and Suganuma A. (1977) Settling velocity of a permeable model floc. Chem. Engng. Sci. 32, 445-447.

Mitani T., Unno H. and Akekata T. (1983) A structure model for activated sludge flocs. Japan Water Res. 6, 65-79.

Namer J. and Ganczarczyk J. J. (1993) Settling properties of digested sludge particle aggregates. Water Res. 27, 1285-1294.

Neale G., Epstein N. and Nader W. (1973) Creeping flow relative to permeable spheres. Chem. Engng. Sci. 28, 1865-1874.

Payatakes A. C. and Dassios G. (1987) Creeping flow around and through a permeable sphere with constant velocity toward a solid wall. Chem. Engng. Commun. 58, $119-138$.

Tambo N. and Watanabe Y. (1979) Physical characteristics of flocs-I. The floc density function and aluminum floc. Water Res. 13, 409-419.

Veerapaneni S. and Wiesner M. R. (1996) Hydrodynamics of fractal aggregates with radially varying permeability. J. Colloid Interf. Sci. 177, 45-57.

Wu R. M., Feng W. H., Tsai I. H. and Lee D. J. (1998) An estimate of waste activated sludge floc permeability: A novel hydrodynamic approach. Water Environ. Res. 70, 1258-1264.

Wu R. M. and Lee D. J. (1998a) Hydrodynamic drag force exerted on a moving floc and its implication to freesettling tests. Water Res. 32, 760-768.

Wu R. M. and Lee D. J. (1998b) Hydrodynamic drag force exerted on a highly porous sphere moving towards an impermeable plate. Chem. Engng. Sci. 53, 3571-3578.

Wu R. M. and Lee D. J. (1999) Highly porous sphere moving through centerline of circular tube filled with Newtonian fluid. Chem. Engng. Sci. 54, 5717-5724.

Wu R. M., Tsou G. W., Lee D. J. (1999) Hydrodynamic response of highly porous sphere. Proceedings of the APCChE, Seoul, Korea.

Zlatanovski T. (1999) Axisymmetric creeping flow past a porous prolate spheroidal particle using the Brinkman model. Quar. J. Mech. Appl. Math. 52, 111-126. 